TITLE:

\title{
Broadening of the $x$-ray emission line due to the instrumental function of the double-crystal spectrometer
}

\section{AUTHOR(S):}

Tochio, T; Ito, Y; Omote, K

\section{CITATION:}

Tochio, T ... [et al]. Broadening of the x-ray emission line due to the instrumental function of the double-crystal spectrometer. PHYSICAL REVIEW A 2002, 65(4): 042502.

ISSUE DATE:

2002-04

URL:

http://hdl.handle.net/2433/50397

RIGHT:

Copyright 2002 American Physical Society 
PHYSICAL REVIEW A, VOLUME 65, 042502

\title{
Broadening of the $x$-ray emission line due to the instrumental function of the double-crystal spectrometer
}

\author{
T. Tochio* and Y. Ito \\ Institute for Chemical Research, Kyoto University, Uji, Kyoto 611-0011, Japan \\ K. Omote \\ X-ray Research Laboratory, Rigaku Co., 3-9-12 Matsubara, Akishima, Tokyo 196-8666, Japan
}

(Received 21 May 2001; published 14 March 2002)

\begin{abstract}
The influence of the instrumental function on the $\mathrm{Cu} K \alpha_{1}$ emission line was investigated for the case of a double-crystal spectrometer. The magnitude of broadening for both $\mathrm{Si}(220)$ and $\mathrm{Si}(440)$ was calculated for a Lorentzian emission line with the width of $1-5 \mathrm{eV}$; the broadening for $\mathrm{Si}(220)$ is $0.12-0.18 \mathrm{eV}$ while that for $\mathrm{Si}(440)$ is only $0.015-0.043 \mathrm{eV}$. The former is too large to be neglected, so the correction for the instrumental function is important. The spectrum affected by the instrumental function seems to keep the shape of Lorentzian though its width is larger. The fact indicates that the Lorentzian fitting analysis is effective if the appropriate correction for width is done.
\end{abstract}

DOI: 10.1103/PhysRevA.65.042502

PACS number(s): 32.30.Rj, 39.30.+w, 07.85.Nc

\section{INTRODUCTION}

The double-crystal spectrometer is one of the most valuable tools for the measurement of the $\mathrm{x}$-ray emission spectrum, and many investigations have been performed with this type of spectrometer so far. In spite of the frequent use of the double-crystal spectrometer, the influence of its instrumental function still remains uncertain. The contribution of the instrumental function of the double-crystal spectrometer is usually considered to be so small that it is often neglected. But the measurement with different crystal planes gives different observed widths as we shall show later. This fact indicates that the proper correction is necessary when we discuss the linewidth with precision of a few tenth $\mathrm{eV}$ or more. In 1954, with confirmation in his subsequent work in 1963, Brogren derived one formula for estimating the true linewidth from his measurements $[1,2]$. His formula was as follows:

$$
W_{T}=W_{0}-W_{C} .
$$

$W_{T}, W_{0}$, and $W_{C}$ mean the true width of the line, the width of the observed emission line, and the width of the rocking curve of the employed crystal in $(+-)$ position, respectively. All widths referred to half-maximum intensity. His formula seems valid because the true width of a certain line derived from his measurements utilizing Eq. (1) is consistent when the plane for diffraction changes. But the difference between the measured width and the true width is in fact, as we shall show later in the present work, usually smaller than the width of the rocking curve of the crystal employed. As for the general formulation for the instrumental function of the double-crystal spectrometer, it was precisely described by Azaroff in 1974 referring even to the tilt of the crystal and the size of the detector [3].

\footnotetext{
*Author to whom correspondence should be addressed.
}

In the present work we have simulated the influence of the instrumental function of the double-crystal spectrometer on the emission spectrum to estimate to what extent the instrumental function practically affects the spectrum. The magnitude of the influence seems to greatly depend on both the wavelength investigated and the plane of the crystal employed. The simulation is applied to the case for radiation having the energy of $\mathrm{Cu} K \alpha_{1}(8048.1 \mathrm{eV})$ observed with $\mathrm{Si}(220)$ and $\mathrm{Si}(440)$.

\section{CALCULATION}

\section{A. Geometry}

The geometry of the system for the double-crystal spectrometer is shown in Fig. 1. In the following, the tilts of the two crystals were not taken into consideration because they are unknown so they cannot be taken into calculation. The radiation emitted from the sample goes into the first crystal after it passes through the Soller slit, which restricts the vertical divergence. The glancing angle of this radiation for the first crystal, denoted by $\eta_{1}$, is given by

$$
\eta_{1}=\frac{\pi}{2}-\arccos (\cos \varphi \sin \theta) \equiv \eta(\theta, \varphi) .
$$

Here $\eta$ is a function that calculates the glancing angle from two arguments. The first argument $\theta$ means the angle between the projection of the radiation on the horizontal plane $[(x, y)$ plane $]$ and the crystal plane for reflection. The second argument $\varphi$ is the angle between the radiation and the horizontal plane. When the positions of the two crystals are for the Bragg angle of $\beta$, the glancing angle for the second crystal is expressed as follows:

$$
\eta_{2}=\eta(2 \beta-\theta, \varphi)=\frac{\pi}{2}-\arccos [\cos \varphi \sin (2 \beta-\theta)] .
$$

Assuming that the radiation emitted from the sample is isotropic, as is the case for $K$ emission spectrum induced by the 


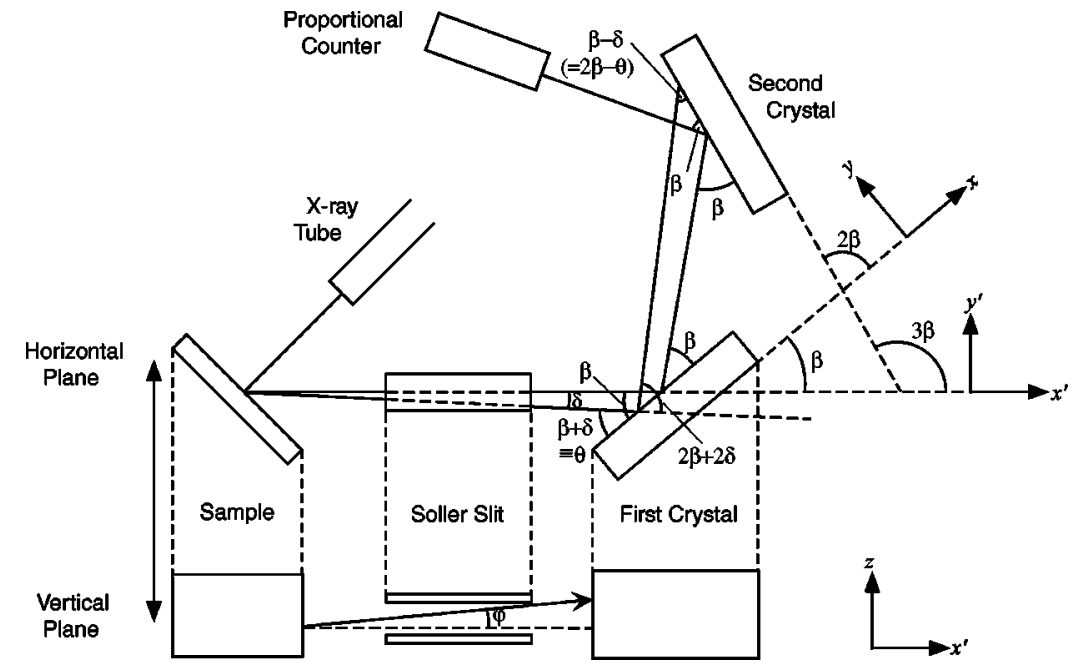

FIG. 1. Schematic diagram for double-crystal spectrometer. secondary excitation, the intensity of the radiation depends only on energy. In this paper, the distribution of the intensity on the energy of radiation is denoted by $F(E)$. The slit function described in detail later is denoted by $S(\varphi)$. If the window of the detector is so large that all the reflected beam from the second crystal could be caught, then $I(\beta)$, the intensity with the crystal positions at the Bragg angle $\beta$, is

$$
\begin{aligned}
I(\beta)= & \int_{0}^{\infty} \int_{-\varphi_{0}}^{\varphi_{0}} \int_{0}^{\pi / 2} F(E) S(\varphi) R\left(E, \eta_{1}\right) \\
& \times R\left(E, \eta_{2}\right) d E d \varphi d \theta,
\end{aligned}
$$

where $R(E, \eta)$ is the rocking curve for the radiation having the energy of $E$ and $\varphi_{0}$ is the maximum value of $\varphi$, which is limited by the Soller slit. What we observe in our measurement is $I(\beta)$ at each point of $\beta$. The spectrometer we used is equipped with a goniometer that makes it possible to change $\beta$ by steps of $0.01^{\circ}, 0.005^{\circ}, 0.002^{\circ}, 0.001^{\circ}$, and $0.0005^{\circ}$. In the rest of this section, the slit function and rocking curve used in the calculation are briefly presented.

\section{Slit function}

The Soller slit is used to restrict the vertical divergence in the spectrometer. The interval of each layer, denoted by $w$, is $1 \mathrm{~mm}$ and the length of this Soller slit, denoted by $\ell$, is 100 $\mathrm{mm}$ (see Fig. 2). Each layer corresponds to a pair of slits that are separated by a distance $\ell$ and whose widths are both $w$. In that case, under the condition that $w / \ell$ is so small that we can approximate $\tan w / \ell$ to $w / \ell$, the slit function $S(\varphi)$ is given by

$$
S(\varphi)=\left\{\begin{array}{cc}
1-\frac{\ell}{w}|\varphi| & \left(|\varphi| \leqslant \frac{w}{\ell} \equiv \varphi_{0}\right) \\
0 & \left(|\varphi|>\varphi_{0}\right) .
\end{array}\right.
$$

The Soller slit used gives $\varphi_{0}$ of 2062.6", which raises the divergence of the glancing angle by $4.52^{\prime \prime}$ and $13.88^{\prime \prime}$ for $\mathrm{Si}(220)$ and $\mathrm{Si}(440)$, respectively, at energy of $\mathrm{Cu} K \alpha_{1}$ $(8048.1 \mathrm{eV})$. Thus the vertical divergence leads to fairly small divergence of the glancing angle while the horizontal divergence causes the divergence of the glancing angle almost of its magnitude. The strong point of the double-crystal spectrometer lies in the fact that the horizontal divergence, which dominantly affects glacing angle, diminishes due to the second crystal. The vertical divergence cannot be restricted by the second crystal because the sum of $\eta_{1}$ and $\eta_{2}$ is not $2 \beta$ when $\varphi$ is not zero.

\section{Rocking curve}

We calculated the rocking curve of $\mathrm{Si}(220)$ and $\mathrm{Si}(440)$ at the energy of $\mathrm{Cu} K \alpha_{1}(8048.1 \mathrm{eV})$ on the basis of dynamical theory with the effect of absorption described by Zachariasen [4]. The effect of absorption appears in the rocking curve as the asymmetric shape. Reflectivity depends on the polarization of incident beam and rocking curves are usually calculated for two specific cases, that is, normal polarization and parallel polarization. In the simulation, where it is assumed that the incident beam is unpolarized, the average of the rocking curve for normal polarization and that for parallel polarization was adopted as $R(E, \eta)$ in Eq. (4). This is based on the assumption that there is no relation in phase between the reflection on the first crystal and that on the second crystal. Besides, the rocking curve depends on the energy of the

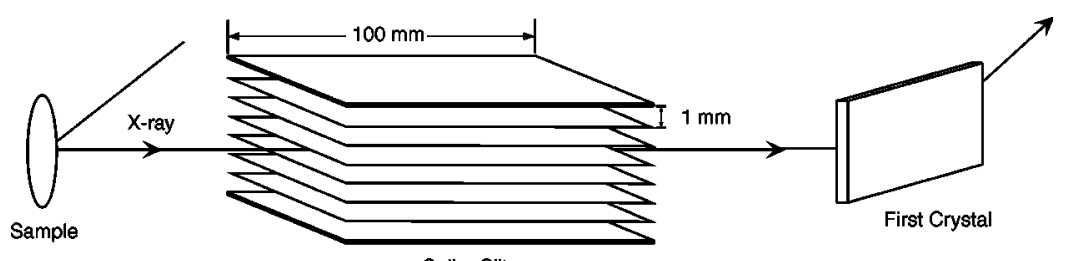

042502-2
FIG. 2. Soller slit that we employed for our measurement $(\ell=100 \mathrm{~mm}, w=1 \mathrm{~mm})$. 

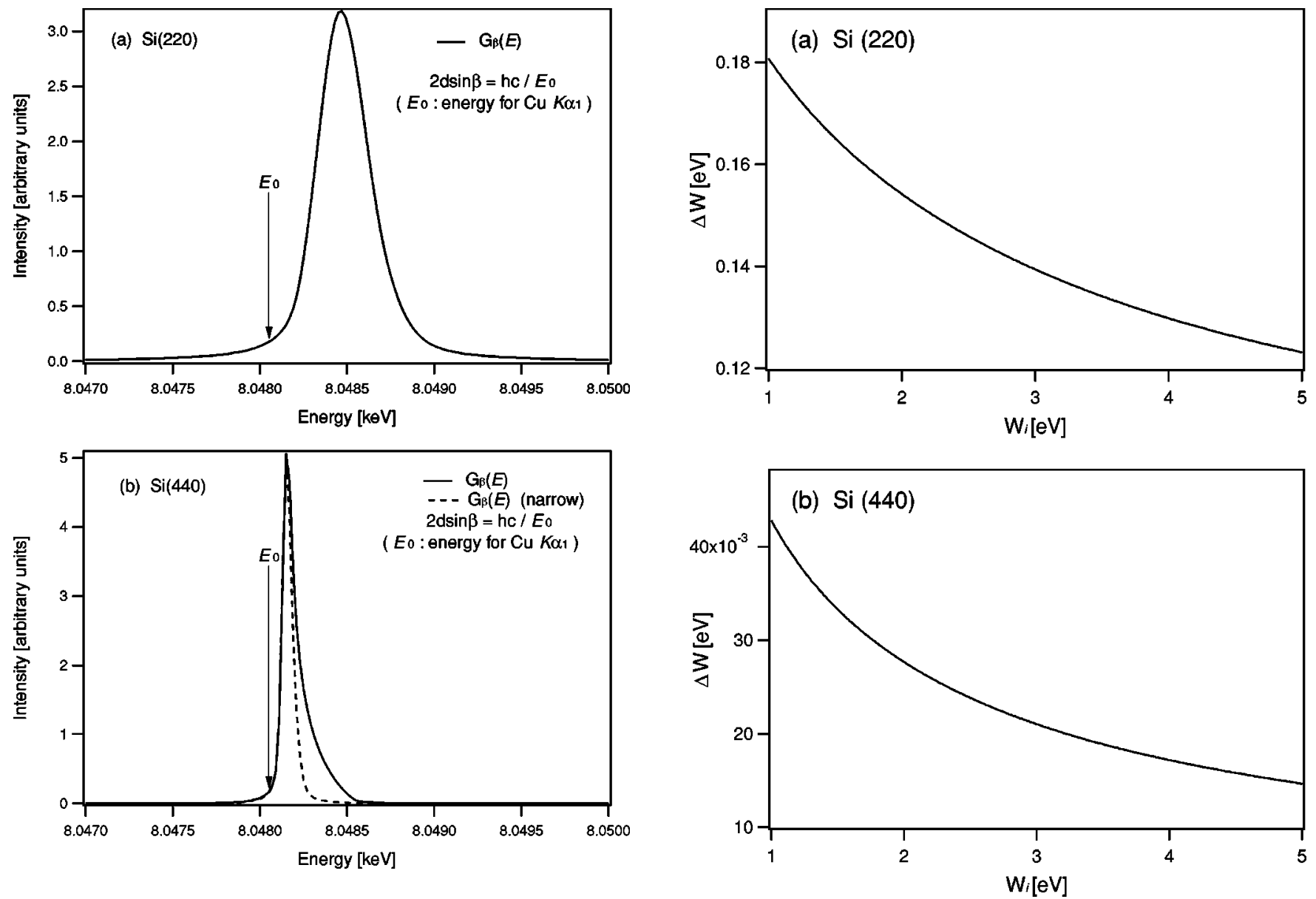

FIG. 3. (a) $G_{\beta}$ (E) for $\mathrm{Si}(220)$. (b) $G_{\beta}(E)$ for $\mathrm{Si}(440)$. [The solid curve shows $G_{\beta}(E)$ with the slit we employed, and the dashed curve shows that with the narrower slit $(\ell=100 \mathrm{~mm}, w$ $=0.5 \mathrm{~mm})$.]

radiation. But the change of the rocking curve in the region for energy in the calculation $\left[\left(E_{0}-12.5\right) \mathrm{eV}\right.$ to $\left(E_{0}\right.$ $+12.5) \mathrm{eV} ; E_{0}$ means the energy investigated] is so small that we are allowed to use a common rocking curve (the rocking curve at $E_{0}$ is likely the best) at any energy. We calculated the rocking curve at the step of $1 \mathrm{eV}$ in the region of $\left(E_{0}-25\right) \mathrm{eV}$ to $\left(E_{0}+25\right) \mathrm{eV}$. In the integration with respect to $\varphi$ and $\theta$, the obtained rocking curve for the energy closest to $E$ was used with the shift of $\eta$ by $\left(\theta_{B_{m}}-\theta_{B}\right)$ in order to improve the precision of the calculation. Here $m$ is an integer between -25 and 25. $\theta_{B_{m}}$ and $\theta_{B}$ correspond to the Bragg angle for the energy of $E_{m}\left[E_{m}=\left(E_{0}+m\right) \mathrm{eV}\right]$ and for the energy investigated, respectively. For example, $R\left(E_{m}, \eta+\theta_{B_{m}}-\theta_{B}\right)$ was employed to give the value of $R(E, \eta)$.

\section{B. Computation}

In the procedure of calculating $I(\beta)$, the integration with respect to $\varphi$ and $\theta$ was done to get $G_{\beta}(E)$ for every position, $\beta$ of two crystals, which is defined as

FIG. 4. Dependence of $\Delta W$ on $W_{i}$ for (a) $\mathrm{Si}(220)$ and (b) $\mathrm{Si}(440)$.

$$
G_{\beta}(E)=\int_{-\varphi_{0}}^{\varphi_{0}} \int_{0}^{\pi / 2} S(\varphi) R\left(E, \eta_{1}\right) R\left(E, \eta_{2}\right) d \varphi d \theta
$$

where $G_{\beta}(E)$ corresponds to the window function on energy $E$ of the spectrometer. To shorten the computation, the range of $\theta$ was reduced to such an extent that almost all the points at which the reflectivity on the first crystal $\left[R\left(E, \eta_{1}\right)\right]$ is less than $10^{-3}$ were excluded. $G_{\beta}(E)$ for $\beta$ satisfying the Bragg condition for the energy of $\mathrm{Cu} K \alpha_{1}$ is shown in Figs. 3(a) and 3(b). It is easily seen that $G_{\beta}(E)$ for $\operatorname{Si}(440)$ is rather asymmetric and has its tail on the high-energy side. $G_{\beta}(E)$ with a narrower vertical Soller slit $(\ell=100 \mathrm{~mm}, w$ $=0.5 \mathrm{~mm}$ ) shows that this is due to the comparatively wider dispersion on the lower side of glancing angle $\eta$ that makes the contribution from the radiation having higher energy larger [see Fig. 3(b)]. In the present work, the step angle of $\beta$ in the simulation for $\mathrm{Si}(220)$ was 4 " while that for $\operatorname{Si}(440)$ was $8^{\prime \prime}$.

$I_{\beta}(E)$ was obtained by integrating $F(E) G_{\beta}(E)$ with respect to $E$ for each $\beta$. F(E) was defined only for the energy between $\left(E_{0}-12.5\right) \mathrm{eV}$ and $\left(E_{0}+12.5\right) \mathrm{eV}$ and the integration of $F(E) G_{\beta}(E)$ with respect to the energy were only performed on this range. We substituted Lorentzian [denoted by $\left.L_{i}(E)\right]$ for $F(E)$ in Eq. (4). The width of $L_{i}(E)$ was 

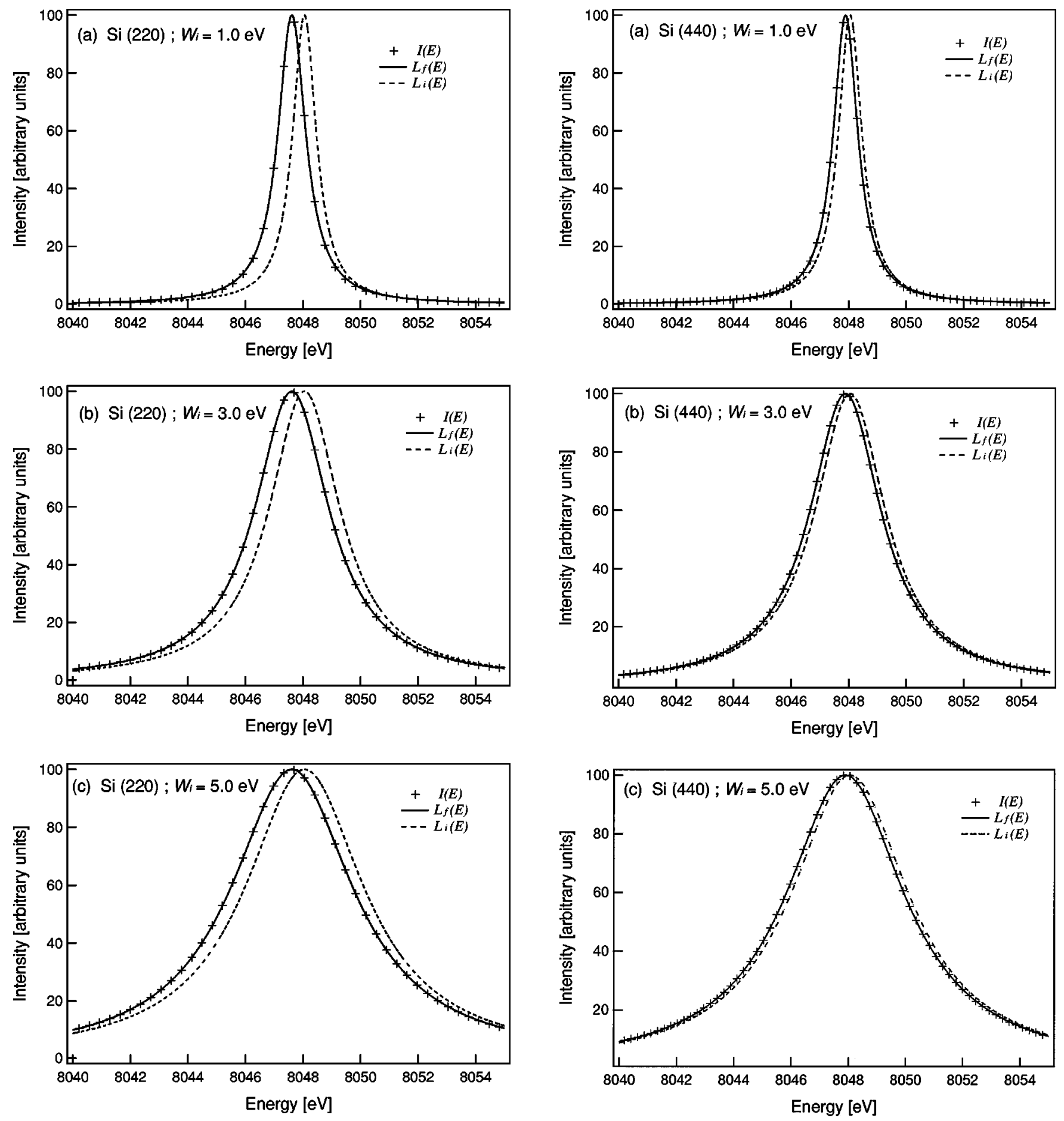

FIG. 5. Change in profile of original Lorentzian with widths of (a) $1.0 \mathrm{eV}$, (b) $3.0 \mathrm{eV}$, (c) $5.0 \mathrm{eV}$ for $\mathrm{Si}(220)$.

varied in the range of 1-5 eV. In addition, to see how much the shape of $I(E)$ is deviated from Lorentzian, we fitted a Lorentzian [denoted by $\left.L_{f}(E)\right]$ to $I(E)$, which is obtained from $I(\beta)$ by converting $\beta$ into the energy that satisfies the Bragg condition, $2 d \sin \beta=h c / E$. We examined the broadening [by comparing the width of $L_{i}(E)$ and that of $\left.L_{f}(E)\right]$ and how much each point of $I(E)$ is deviated from $L_{f}(E)$. It should be noted that several points, which are close to the edges, were eliminated in the fit procedure because the

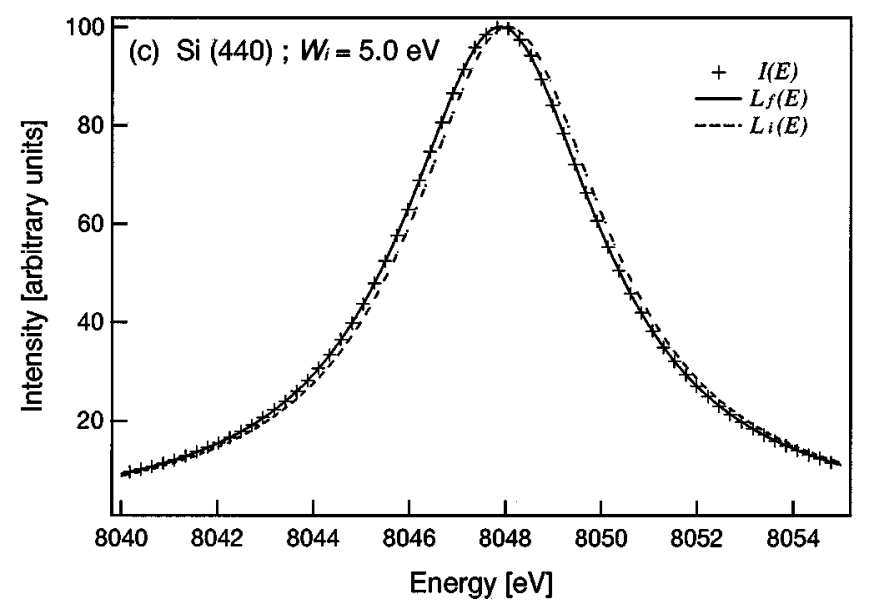

FIG. 6. Change in profile of original Lorentzian with widths of (a) $1.0 \mathrm{eV}$, (b) $3.0 \mathrm{eV}$, (c) $5.0 \mathrm{eV}$ for $\mathrm{Si}(440)$.

points near the edges are subjected to the influence of missing the curve in the further region.

\section{RESULTS AND DISCUSSION}

Figures 4(a) and 4(b) shows the broadening $\Delta W\left(\equiv W_{f}\right.$ $-W_{i}$ ) determined by the calculation. It is easily seen that the larger width of $L_{i}(E)$ leads the smaller broadening both for $\mathrm{Si}(220)$ and for $\mathrm{Si}(440)$. In the region of $1-5 \mathrm{eV}$ for $W_{i}$, the 

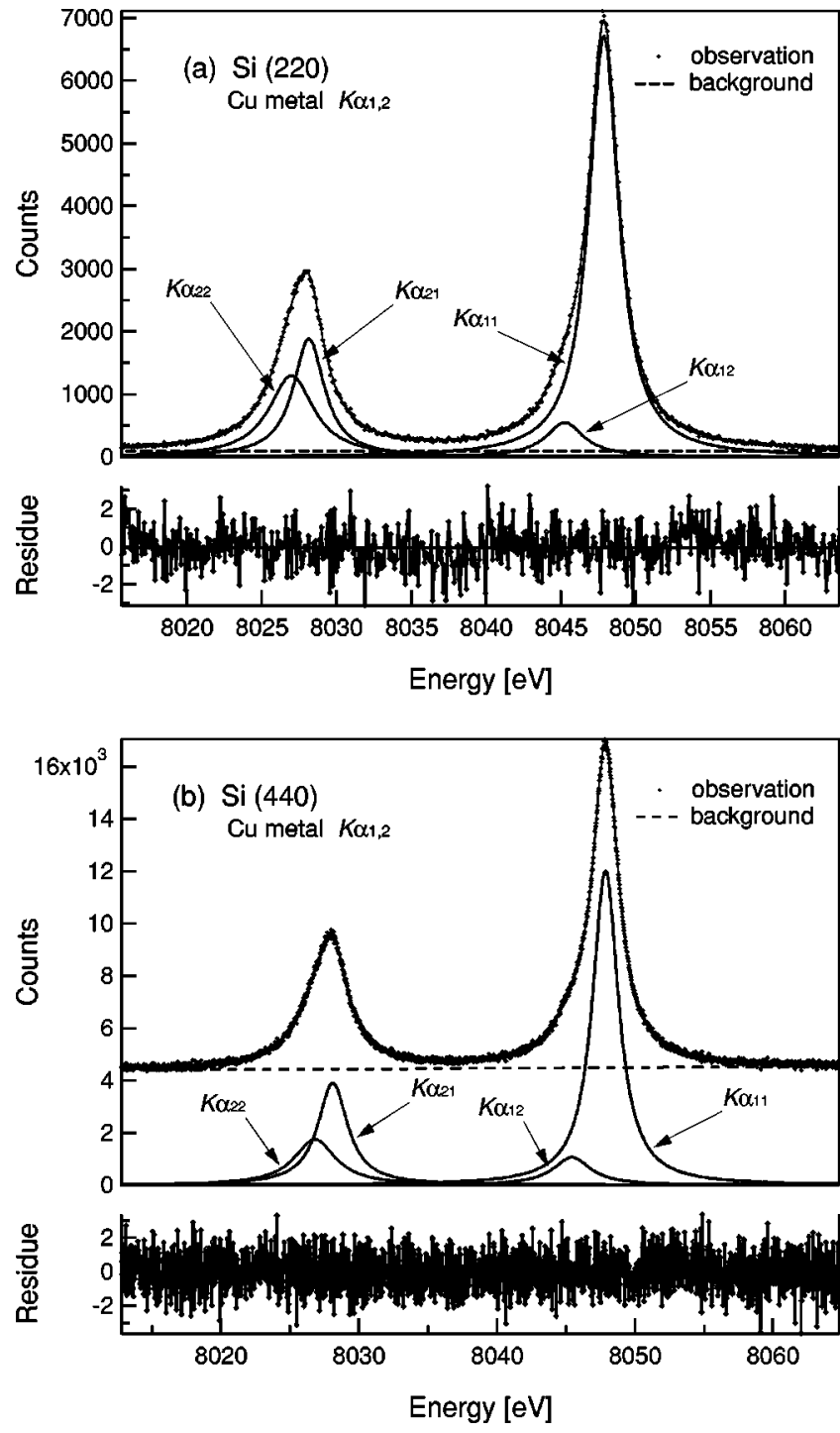

FIG. 7. Spectra of $\mathrm{Cu} K \alpha$ measured using double-crystal spectrometer with double crystal of (a) $\mathrm{Si}(220)$ and (b) $\mathrm{Si}(440)$.

magnitude of broadening is in the range $0.12-0.18 \mathrm{eV}$ and $0.015-0.043 \mathrm{eV}$ for $\mathrm{Si}(220)$ and $\mathrm{Si}(440)$, respectively. On the other hand the width of the rocking curve (average of normal and parallel polarization) for $\mathrm{Si}(220)$ and $\mathrm{Si}(440)$ are $0.46 \mathrm{eV}$ and $0.07 \mathrm{eV}$, respectively. This indicates that the magnitude of broadening is much smaller than the width of the rocking curve for both cases and the correction using Eq. (1) may underestimate the true width. The broadening for $\mathrm{Si}(440)$ is so small that the width without any correction is almost the same as the true width, though for $\operatorname{Si}(220)$ this is not the case.
The deviation of $I(E)$ from $L_{f}(E)$ can be seen in Figs. 5(a) $-5(\mathrm{c})$ for $\mathrm{Si}(220)$ and Figs. 6(a) $-6(\mathrm{c})$ for $\mathrm{Si}(440)$, respectively. It appears that all the points of $I(E)$ are very close to $L_{f}(E)$ for all cases. This means that it may be allowed to fit Lorentzian to the spectrum observed.

For reference, the measured $\mathrm{Cu} K \alpha$ spectra are shown in Fig. 7. Please note that the following discussion has no relation with various phenomena involved in the $\mathrm{Cu} K \alpha$ spectra. Both spectra were measured using the same double-crystal spectrometer, but with $\operatorname{Si}(220)$ for one and $\operatorname{Si}(440)$ for the other. We fitted four Lorentzians to these spectra and compared the widths of the fitted Lorentzians. In the measurement, a $\mathrm{Rh}$ x-ray tube was used for exciting $\mathrm{Cu} K$ radiation. It was operated at $40 \mathrm{kV}, 70 \mathrm{~mA}$. The results for $K \alpha_{11}$, which are considered to be most reliable, were compared. As can be seen from Table I, the width of the fitted Lorentzian $W_{f}$ for $K \alpha_{11}$ is $2.454 \mathrm{eV}$ and the calculation gives $2.305 \mathrm{eV}$ for $W_{i}$ in the case of $\mathrm{Si}(220)$. Similarly, in the case of $\mathrm{Si}(440)$, the calculation gives $2.254 \mathrm{eV}$ for $W_{i}$ when $W_{f}$ is $2.280 \mathrm{eV}$. For comparison, we tabulated, with ours, the true widths of $\mathrm{Cu} K \alpha_{11}$ obtained by other researchers $[5,6]$ using a double-crystal spectrometer (Table II). The deviation among them is less than $0.07 \mathrm{eV}$. Considering the fact that the difference between the widths deduced from the two independent measurements with the same reflection $\mathrm{Si}(333)$ is about $0.06 \mathrm{eV}$, the values tabulated seem close to one another.

One of the ways to evaluate the validity of our method is comparing our two corrected widths. These should be the same ideally. However, as can be seen from Table II, there still remains the difference of $0.05 \mathrm{eV}$. This difference might not be attributed only to the error in calculation but to that in measurement, which includes the aberrant alignment of the optical system. In either case, it is necessary to investigate a specific line using the same spectrometer with various conditions and see how the corrected widths agree with one another. It is desirable that the line to be investigated is intense and symmetric.

\section{CONCLUSION}

We investigated the influence of the instrumental function of a double-crystal spectrometer by calculating the broadening of emission line having the energy corresponding to $\mathrm{Cu}$ $K \alpha_{1}$. The result was that the contribution of the instrumental function to the emission spectrum cannot be neglected and that it is possible to estimate its magnitude with calculation. Our results also show that the broadening of $\mathrm{Cu} K \alpha_{11}$ for $\mathrm{Si}(220)$ and $\mathrm{Si}(440)$ are roughly $0.149 \mathrm{eV}$ and $0.026 \mathrm{eV}$,

TABLE I. Widths of four fitted Lorentzians for $\mathrm{Cu} K \alpha_{1,2}$ observed with the crystal of $\mathrm{Si}(220)$ and $\mathrm{Si}(440)$. All values are in units of $\mathrm{eV}$. The values in parentheses are errors in the Lorentzian fitting.

\begin{tabular}{ccccc}
\hline Crystal & $K \alpha_{11}$ & $K \alpha_{12}$ & $K \alpha_{21}$ & $K \alpha_{22}$ \\
\hline $\operatorname{Si}(220)$ & $2.454(0.013)$ & $3.071(0.113)$ & $2.602(0.091)$ & $3.571(0.078)$ \\
$\operatorname{Si}(440)$ & $2.280(0.008)$ & $3.290(0.093)$ & $2.612(0.061)$ & $3.661(0.083)$ \\
\hline \hline
\end{tabular}


TABLE II. True widths (corrected widths) of $\mathrm{Cu} K \alpha_{11}$ obtained by using a double-crystal spectrometer. All values are in units of $\mathrm{eV}$.

\begin{tabular}{lcccc}
\hline \hline & Härtwig et al. [5] & Deutsch et al. [6] & \multicolumn{2}{c}{ Present work } \\
\hline Reflection & $\operatorname{Si}(333)$ & $\mathrm{Si}(333)$ & $\mathrm{Si}(220)$ & $\mathrm{Si}(440)$ \\
Full width at half maximum & 2.24 & 2.298 & 2.305 & 2.254 \\
\hline \hline
\end{tabular}

respectively. The former cannot be neglected for the observed linewidth and the correction for the broadening is important. As to the change in profile, it seems that the curves affected by the instrumental function are almost Lorentzian with wider width than that of the original. In this case, we can effectively use the Lorentzian fitting with the correction for the width. It is necessary to apply our calculation to various lines with various crystal planes.

\section{ACKNOWLEDGMENTS}

Our measurement was performed at Hyogo Prefectural Institute of Industrial Research. One of the authors (T.T.) thanks M. Motoyama and T. Kaneyoshi for their support with our measurement, and also A. M. Vlaicu for his help in studying the instrumental function of the double-crystal spectrometer.
[1] G. Brogen, Ark. Fys. 8, 391 (1954).

[2] G. Brogen, Ark. Fys. 23, 219 (1963).

[3] L. V. Azaroff, X-Ray Spectroscopy (McGraw-Hill, New York, 1974).

[4] W. H. Zachariasen, Theory of X-Ray Diffraction in Crystals
(Wiley, New York, 1945).

[5] J. Härtwig, G. Hölzer, J. Wolf, and E. Förster, J. Appl. Crystallogr. 26, 539 (1993).

[6] M. Deutsch, G. Hölzer, J. Härtwig, J. Wolf, M. Fritsch, and E. Förster, Phys. Rev. A 51, 283 (1995). 